\title{
A STUDY OF POSITRON PROPERTIES IN QUARTZ CRYSTALS AND SYNTHETIC SILICA GLASS
}

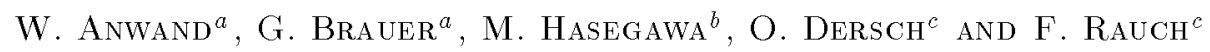 \\ ${ }^{a}$ Institut für Ionenstrahlphysik und Materialforschung, Forschungszentrum Rossendorf \\ Postfach 5101 19, 01314 Dresden, Germany \\ ${ }^{b}$ Institute for Materials Research, Tohoku University, Sendai 980-8577, Japan \\ ${ }^{c}$ Institut für Kernphysik, Universität Frankfurt \\ August-Euler-Str. 6, 60486 Frankfurt/Main, Germany
}

\begin{abstract}
The monoenergetic positron beamline "SPONSOR" at Rossendorf has been used to investigate the positron behaviour in a naturally grown Brasilian quartz, two synthetic quartz crystals of different origin, and synthetic silica glass. The measurements allow us to obtain the positron diffusion length of free positrons and Bloch para-positronium, if formed, in these materials. In addition, hydrothermal treatment of a synthetic quartz has been used to introduce hydrogen into the crystal up to a certain depth. The presence of hydrogen is found to influence the formation of para-positronium. The depth distribution of hydrogen has been measured independently by nuclear reaction analysis, and will be discussed in comparison with the results deduced from the positron studies.
\end{abstract}

PACS numbers: $78.70 . \mathrm{Bj}$

\section{Introduction}

$\mathrm{SiO}_{2}$ in solid form (amorphous or crystalline) represents an interesting material to study the properties of positronium $(\mathrm{Ps})$ [1]. Positrons are a very good tool to study different states of order, or bond changes, in amorphous silica glass and $\mathrm{SiO}_{2}[2-4]$. Especially, the estimation of the crystallinity and of fundamental physical parameters, as the diffusion lengths of positrons and Ps, is possible using the positron beam technique.

\section{Experimental}

Five samples have been investigated which can be shortly characterized as follows:

- Brasilian quartz: very clean untreated, naturally grown, density: $2.65 \mathrm{~g} \mathrm{~cm}^{-3}$, 
- synthetic silica glass: produced by spraying of destilled silicontetrachloride into a detonating gas fire, density: $2.21 \mathrm{~g} \mathrm{~cm}^{-3}$,

(both the samples have been studied by positron lifetime spectroscopy earlier [2])

- synthetic alpha-quartz A (Kinseki Co./Japan), density: $2.51 \mathrm{~g} \mathrm{~cm}^{-3}$, $\mathrm{Pt}<0.2, \mathrm{Na}: 0.2, \mathrm{Li}: 0.5, \mathrm{Al}: 1.6 \mathrm{mg} / \mathrm{kg}$,

- two synthetic alpha-quartz B samples (Crystal Co. Berlin/Germany), $\mathrm{Al}<30 \mathrm{ppm}, \mathrm{Fe}<5 \mathrm{ppm}, \mathrm{Na}<10 \mathrm{ppm}, \mathrm{Li}<3 \mathrm{ppm}, \mathrm{H}=20 \mathrm{ppm}$ (determined at Frankfurt/M with nuclear reaction analysis (NRA)), one sample untreated, one sample hydrothermally treated in a closed steel vessel for 5 days at $200^{\circ} \mathrm{C}$ and $1.5 \mathrm{Mpa}$, density: $2.65 \mathrm{~g} \mathrm{~cm}^{-3}$.

The slow positron implantation spectroscopy (SPIS) was carried out at the slow positron beam "SPONSOR" at Rossendorf [5]. The positrons are magnetically guided to the sample and can be accelerated in the energy range from $30 \mathrm{eV}$ to $35 \mathrm{keV}$ in steps of $100 \mathrm{eV}$. The energy resolution of the Ge detector at $511 \mathrm{keV}$ is $(1.09 \pm 0.01) \mathrm{keV} .7 \times 10^{5}$ events per spectrum at each incident positron energy have been accumulated. $\mathrm{H}$ was profiled with the resonant nuclear reaction ${ }^{1} \mathrm{H}\left({ }^{15} \mathrm{~N}, \alpha \gamma\right){ }^{12} \mathrm{C}$. The local hydrogen concentration is proportional to the number of $4.43 \mathrm{MeV} \gamma$-rays produced by the reaction. The depth resolution in quartz is $7 \mathrm{~nm}$ at the sample surface. The measurements were performed with the $7 \mathrm{MV}$ Van de Graaf accelerator of the Institut für Kernphysik (Frankfurt am Main) using a set-up for a high-sensitivity analysis of hydrogen [6].

\section{Results and discussion}

\subsection{Comparison between Brazilian and synthetic alpha-quartz A and synthetic silica glass}

The $S$ versus $E$ plots of Brazilian and synthetic alpha-quartz A and synthetic silica glass are shown in Fig. 1. Differences in the shape of the plots are mainly caused by the formation of Ps in the samples. Brazilian quartz can be considered fully crystalline. Furthermore it is concluded from positron lifetime measurements in [2] that no Ps formation takes places in this quartz. The synthetic silica glass shows an amorphous structure. A pick-off annihilation of $o-\mathrm{Ps}$ of $40.9 \%$ was observed in this material [2]. That means, the third part of this amount is detected as $p$-Ps in the $S(E)$ plot. Assuming the difference in $S_{\text {bulk }}$ between silica glass and Brazilian quartz is mainly caused by the Ps formation and other influences can be neglected, it is possible to calculate the $S$ parameter for the $p$-Ps annihilation $S_{p-P s}$ as follows:

$$
S_{\text {bulk }}^{\text {silica }}=(1-\beta) S_{\text {bulk }}^{\text {Braz. }}+\beta S_{p-\mathrm{Ps}}
$$

with $\beta-p$-Ps fraction, $S_{\text {bulk }}^{\text {silica }}-S_{\text {bulk }}$ of synthetic silica glass, $S_{\text {bulk }}^{\text {Braz. }}-S_{\text {bulk }}$ of Brazilian quartz. With the known $S_{\text {bulk }}^{\text {silica }}, S_{\text {bulk }}^{\text {Braz. }}$ and $\beta=0.136$, the $S$ parameter, $S_{p-\mathrm{Ps}}$, for the $p$-Ps annihilation could be calculated to be 0.9824 . Using formula (1) again for the synthetic quartz A with the known $S_{p-P s}$ it is possible to calculate the $p$-Ps fraction in this sample to be $3.4 \%$. 




Fig. 1. $S$ parameter versus incident positron energy of three different materials.

The local maximum of the $S$ parameter of the synthetic alpha-quartz $A$ at $1.53 \mathrm{keV}$ is caused by the formation of $p$-Ps and its diffusion to the surface of the sample. Ps that has left the sample does not contribute to the $S$ parameter. For this reason, a decrease in the $S$ parameter at lower incident positron energies is observed. This gives the opportunity to estimate the diffusion length of Ps in this material. Assuming, that only the influence of the Ps formation causes the differences between Ps-free Brazilian quartz and synthetic alpha-quartz A and synthetic silica glass, the Ps diffusion length could be calculated.

The procedure for the estimation of the Ps diffusion length can be described as follows: At low incident positron energies $E$ the Ps gets the chance to leave the sample in the case when its diffusion length is longer than its implantation depth. For $E=1.03 \mathrm{keV}$ the difference $\delta S$ of the $S$ parameters between the Ps free Brazilian quartz and the synthetic alpha-quartz A was calculated. Figure 2 shows the difference $\delta S$ versus the incident positron energy $E$ for both synthetic alpha-quartz A and synthetic silica glass. From the measured surface $S$ parameters

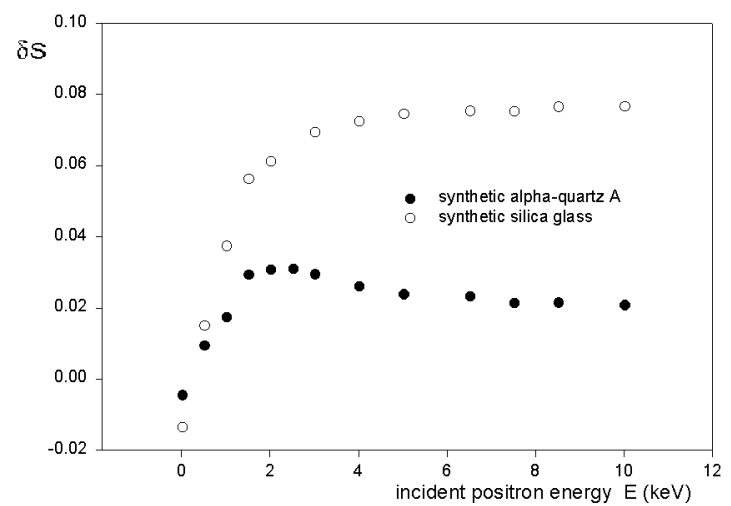

Fig. 2. Differences of the $S$ parameters between Brazilian quartz and synthetic alpha-quartz A and synthetic silica glass. 
$S_{s}$ of both samples it is seen that the surface fraction has only a small influence on the difference $\delta S$ and can be ignored. The contribution of epithermal positrons to the $S$ parameter is assumed to be equal for both samples and disappears in $\delta S$. This results in the following formula:

$$
\delta S=m_{\mathrm{p}} S_{\mathrm{p}}+m_{\mathrm{b}} S_{\mathrm{b}}-n_{\mathrm{b}} S_{\mathrm{b}}
$$

where $S_{\mathrm{p}}$ and $S_{\mathrm{b}}$ are the $S$ parameters of the $\mathrm{Ps}_{\mathrm{s}}$ and the Ps free bulk, $m_{\mathrm{p}}$ and $m_{\mathrm{b}}$ are the fractions of $P s$ and bulk in the sample with Ps formation, $n_{\mathrm{b}}$ is the fraction of the Ps free bulk. Furthermore, one can set $m_{\mathrm{p}} \cong n_{\mathrm{b}}-m_{\mathrm{b}}$. This leads to the following equation:

$$
m_{\mathrm{p}} \cong \delta S /\left(S_{\mathrm{p}}-S_{\mathrm{b}}\right)
$$

The calculated fraction of Ps that contributes to the $S$ parameter at $E=1.03 \mathrm{keV}$ could be estimated using Eq. (3) to be $m_{\mathrm{p}}=0.905$. That means, $9.95 \%$ of the Ps in the synthetic alpha-quartz A leaves the sample at this incident positron energy. Using the Makhovian profile $P(E=1.03 \mathrm{keV}, d)$ and calculating the Ps fraction that is leaving the sample with the model of a spherical segment (Fig. 3), the diffusion length of Ps in synthetic alpha-quartz A could be obtained as $L_{+\mathrm{Ps}}=18 \mathrm{~nm}$ according to

$$
m_{V}=\sum_{i}\left(P\left(E, d_{i}\right)\left(L_{+\mathrm{Ps}}-d_{i}\right)^{2}\left(L_{+\mathrm{Ps}}+d_{i}\right) / 4 L_{+\mathrm{Ps}}\right),
$$

where $m_{V}$ - Ps fraction leaving the sample. The same procedure was done for the synthetic silica glass at an energy of $E=1.53 \mathrm{keV}$ resulting in a Ps diffusion length of $L_{+\mathrm{Ps}}=37 \mathrm{~nm}$. The reason for the higher Ps diffusion length in the amorphous silica glass is obviously the higher amount of free volume in this sample resulting in a higher mobility of Ps.


Fig. 3. Makhovian profile $P(1 \mathrm{keV}, d)$ and the "diffusion sphere" for the calculation of the Ps diffusion length.

The above-mentioned procedure for calculating the Ps diffusion length in the bulk cannot be used for very low incident positron energies. Near the surface the Ps becomes more mobile and its diffusion seems to be directed preferably towards 
the surface as the calculations have shown. Furthermore, the calculations have shown that near the surface of the alpha-quartz A more Ps are formed as in the bulk.

The crystallinity of the synthetic alpha-quartz A can be calculated using the following formula from Ref. [2]:

$$
S^{*}=(1-\alpha) S_{\mathrm{a}}+\alpha S_{\mathrm{c}},
$$

with $\alpha=V_{\mathrm{c}} /\left(V_{\mathrm{a}}+V_{\mathrm{c}}\right), S_{\mathrm{a} / \mathrm{c}}$ means the $S$ parameter of amorphous and crystalline material, $S^{*}$ is the $S$ parameter of partial amorphous material, $V_{\mathrm{a} / \mathrm{c}}$ - the amorphous and crystalline volume fraction, $\alpha$ is the relative fraction of crystalline volume. For the synthetic quartz A we found $\alpha=0.75$. That means $25 \%$ of the whole volume is amorphous.



Fig. 4. Brasilian quartz, VEPFIT results.

The positron diffussion length in the crystalline Brazilian quartz is considered as an important parameter. Figure 4 shows the $S(E)$ measured for the Brazilian quartz and the curve resulting from VEPFIT [7]. The positron diffusion length could be calculated as $L_{\text {+bulk }}=(18 \pm 1) \mathrm{nm}$ and $S_{\text {bulk }}=0.4191$.

It should be mentioned that according to the lifetime results [2] the Brazilian quartz still contains defects which trap positrons. This means that $L_{+}$for defect free material might be still larger than $18 \mathrm{~nm}$ as estimated here.

Because of the strong influence of Ps, especially near the surface, it was impossible to separate the Ps fraction from the part of the positrons and for this reason no positron diffusion lengths could be fitted for quartz with Ps formation.

\subsection{Synthetic alpha-quartz B (Crystal Co., Berlin) and the effect of a hydrothermal treatment}

Concerning the $S(E)$ there are only small differences between synthetic alpha-quartz A and the synthetic alpha-quartz B that has been used as a virgin material for the hydrothermal treatment as seen in Fig. 5. The $S_{\text {bulk }}$ of both samples do not differ too much from each other. For this reason, the crystallinity 




Fig. 5. $S(E)$ of synthetic alpha-quartz B (Crystal Co. Berlin) compared to Brazilian and synthetic alpha-quartz A.

is nearly the same: $\alpha=0.77$. However, at low incident positron energies a considerable higher $S$ parameter was measured. This is an evidence of a shorter Ps diffusion length in this sample. The calculated diffusion length of $L_{+} \mathrm{Ps}=8 \mathrm{~nm}$ supports this fact.

The influence of the hydrothermal treatment on the Ps behaviour is shown in Fig 6. Due to the hydrothermal treatment a $\mathrm{H}$ rich layer below the surface had been created. The $\mathbf{H}$ concentration profile has roughly the shape of the error function complement, decreasing from the surface with depth. The concentration at the surface is 0.9 at.\% and 0.45 at.\% at $50 \mathrm{~nm}$ depth. It is unknown whether the binding state of $\mathrm{H}$ in the quartz is as $\mathrm{H}_{2} \mathrm{O}$ or OH. Probably, both configurations are present. $\mathrm{H}^{+}$is unlikely $[8]$.

It is seen from the $S(E)$ curves in Fig. 6 that the hydrogen does not have an effect on the Ps in the bulk as expected. Immediately below the surface the differences become visible. The calculation of the Ps diffusion length led to the

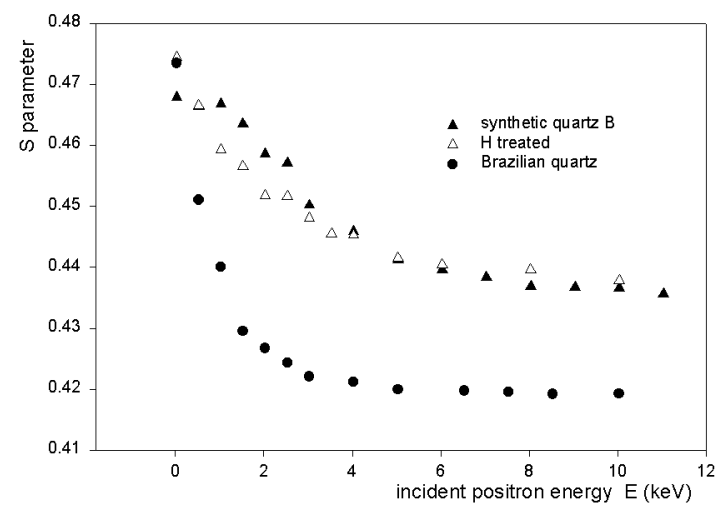

Fig. 6. The influence of $\mathrm{H}^{+}$incorporation on the Ps behaviour in synthetic quartz. 




Fig. 7. Makhovian implantation profiles compared with the $\mathrm{H}$ rich layer in hydrothermally treated synthetic alpha-quartz B.

same value as in the case of the $H$ free sample: $L_{+\mathrm{Ps}}=8 \mathrm{~nm}$. However, the lower $S$ parameter in the range from $1.0 \mathrm{keV}$ to $3.5 \mathrm{keV}$ allows for the conclusion that the hydrogen suppresses partially the formation of Ps by occupying open volume. This leads to a decrease in the $S$ parameter in this range. An impression about the depth depending Ps formation is given in Fig. 7, where the Makhovian implantation profiles of this energy range are presented. From Fig. 6 it follows that at positron implantation energies above $3 \mathrm{keV}$ the difference between the $\mathrm{H}$ treated and untreated quartz disappears. That means, the positron beam measurements lead to a thickness of a $\mathrm{H}$ rich layer of about $70 \mathrm{~nm}$. This finding is in excellent agreement with the NRA data given above.

\section{Conclusions}

It could be demonstrated that it is possible to extract the positronium diffusion length from the SPIS investigations in connection with lifetime measurements. The diffusion length of $18 \mathrm{~nm}$ of the crystalline Brasilian quartz has been calculated. The Ps diffusion length in amorphous silica glass is significantly higher $L_{+\mathrm{Ps}}=37 \mathrm{~nm}$. These results show that the mobility of the Ps becomes higher, its diffusion length increases, if the crystallinity of the quartz decreases.

The presence of $\mathrm{H}$ in synthetic alpha-quartz suppresses partially the formation of Ps. This phenomenon can be explained due to the fact that $\mathrm{H}$ occupies free volume in the quartz that is not available for the Ps formation any more.

\section{References}

[1] M. Hasegawa, M. Saneyasu, M. Tabata, Z. Tang, Y. Nagi, T. Chiba, Y. Ito, Nucl. Instrum. Methods B 166-167, 431 (2000).

[2] G. Braner, G. Boden, A. Balogh, A. Andreeff, Appl. Phys. 16, 231 (1978).

[3] M. Saneeyasu, M. Hasegawa, Z. Tang, M. Tabata, M. Fujinami, Y. Ito, S. Yamaguchi, Mater. Sci. Forum 255-257, 460 (1997). 
[4] M. Fujinami, N.B. Chilton, K. Ishii, Y. Ohki, J. Phys. IV (France) 3, 169 (1993).

[5] W. Anwand, H.-R. Kissener, G. Brauer, Acta Phys. Pol. A 88, 7 (1995).

[6] D. Endisch, H. Sturm, F. Rauch, Nucl. Instrum. Methods B 84, 380 (1994).

[7] A. van Veen, H. Schut, J. de Vries. R.A. Hakvoort, M.R. Ijpma, in: Positron Beams for Solids and Surfaces, Eds. P.J. Schultz, G.R. Massoumi, P.J. Simpson, AIP Conf. Proc., Vol. 218, American Institute of Physics, New York 1990, p. 171.

[8] O. Dersch, F. Rauch, Fresenius J. Anal. Chem. 365, 114 (1999). 